\title{
Plasmonic Nanostructure for Enhanced Light Absorption in Ultrathin Silicon Solar Cells
}

\author{
Jinna He, ${ }^{1,2}$ Chunzhen Fan, ${ }^{1}$ Junqiao Wang, ${ }^{1}$ Yongguang Cheng, ${ }^{1}$ Pei Ding, ${ }^{3}$ and Erjun Liang ${ }^{1}$ \\ ${ }^{1}$ School of Physical Science and Engineering and Key Laboratory of Material Physics of The Ministry of Education of China, \\ Zhengzhou University, Zhengzhou 450052, China \\ ${ }^{2}$ Electric and information Engineering College, Pingdingshan University, Henan Province, Pingdingshan 467000, China \\ ${ }^{3}$ Department of Mathematics and Physics, Zhengzhou Institute of Aeronautical Industry Management, Zhengzhou 450015, China
}

Correspondence should be addressed to Erjun Liang, ejliang@zzu.edu.cn

Received 9 July 2012; Revised 18 September 2012; Accepted 24 September 2012

Academic Editor: Pavel A. Belov

Copyright (c) 2012 Jinna He et al. This is an open access article distributed under the Creative Commons Attribution License, which permits unrestricted use, distribution, and reproduction in any medium, provided the original work is properly cited.

\begin{abstract}
The performances of thin film solar cells are considerably limited by the low light absorption. Plasmonic nanostructures have been introduced in the thin film solar cells as a possible solution around this issue in recent years. Here, we propose a solar cell design, in which an ultrathin Si film covered by a periodic array of Ag strips is placed on a metallic nanograting substrate. The simulation results demonstrate that the designed structure gives rise to $170 \%$ light absorption enhancement over the full solar spectrum with respect to the bared Si thin film. The excited multiple resonant modes, including optical waveguide modes within the Si layer, localized surface plasmon resonance (LSPR) of Ag stripes, and surface plasmon polaritons (SPP) arising from the bottom grating, and the coupling effect between LSPR and SPP modes through an optimization of the array periods are considered to contribute to the significant absorption enhancement. This plasmonic solar cell design paves a promising way to increase light absorption for thin film solar cell applications.
\end{abstract}

\section{Introduction}

The low conversion efficiencies and high production costs have been the major difficulties facing photovoltaic technology. For solar cells based on bulk crystalline silicon, around $40 \%$ of a solar cell module's price comes from the silicon (Si) materials and its processing costs. To reduce the costs, thin film solar cells with an active layer thickness of about 1 to $2 \mu \mathrm{m}$ are desired. Thin film solar cells with the thickness of material film smaller than the carrier diffusion length can also reduce carrier recombination and improve carrier collection efficiency in bulk recombination-dominated semiconductors. In addition, a significant reduction of the active materials enables some scare semiconductor materials such as Te and In to be used in a large scale. However, the performance of all thin film solar cells is limited by the poor light absorption due to the reduced absorber thickness. For example, the indirect band gap semiconductor Si material has poor absorption to near-band gap light, where the absorption length is larger than $300 \mu \mathrm{m}$. Therefore, light trapping schemes are essential for the design of ultrathin solar cells with improved absorption.

In the past years, many light trapping techniques have been investigated for solar cell applications. A typical example is the use of micron-size pyramidal surface textures [1]. However, such textures are not suitable for thin film solar cells due to large texturing size with respect to the film thickness. Recently, the concept of plasmonic solar cells, that is, the combination of plasmonics and photovoltaic fields, has been proposed to improve light absorption [24]. Metallic nanostructures engineered within the solar cell geometry enable concentrating and folding light into the ultrathin active layer, and thereby increase light absorption. For example, Au or Ag nanoparticles placed on the top surface of solar cells can act as scattering elements to couple light into the absorber layer through effectively increasing the optical path length [5-8]. Over 30\% enhancement in photocurrent has been demonstrated experimentally in a $1.25 \mu \mathrm{m}$ thick silicon-on-insulator by utilizing this approach [8]. The dependences of incoupling efficiency on 
nanoparticle shape and size have also been discussed in detail [9]. Furthermore, the strong near fields resulting from the LSPRs excitations of metallic strips on the top of the active layer can effectively trap light into the absorber layer and lead to an enhancement of about $43 \%$ in short circuit currents [10]. More recently, a kind of novel thin film solar cell design of introducing several metallic patterned back contacts has been investigated widely [11-15]. These patterned back contacts can couple the sunlight into SPP mode propagating along the metal/semiconductor interface and waveguide mode within the absorber layer, leading to about 30\% broadband absorption enhancement over the solar spectrum when compared to the bared thin film cells [11].

Since the excitation of plasmon resonances can capture and trap the sunlight into the active layer and increase absorption strength, multiple plasmon resonances are desired for the thin film solar cell with superior performance. Previous designs of plasmonic solar cells are mostly based on placing one- or two-dimensional metallic nanoparticle arrays on the top or buried inside the active layer $[5-8,16-$ 18], or introducing several metallic nanogratings, such as the nanohole, hexagonal or triangular structures [12, 19, 20] at the bottom of active layer as the patterned back contacts for improving light absorption. The common disadvantages associated with those efforts are that LSPR or SPP modes are excited individually only by utilizing the metallic nanoparticles or nanograting structures. In this work, we propose an ultrathin solar cell design, which consists of a periodic array of Ag strips on a silica-coated Si film supported by a metallic grating substrate, as shown in Figure 1. This design not only takes advantage of LSPR excited in metal strips on the top, but also can couple sunlight to multiple SPP modes at metal/semiconductor interface by the coupling of the bottom nanograting. Furthermore, the coupling effects between the LSPR and SPP modes can be achieved by tuning the array periods for further increasing light absorption inside the active layer. A remarkable absorption enhancement of about $170 \%$ with a broad period range is obtained over the full solar spectrum when compared with the case of metallic nanostructures absence (i.e., the bared thin film cells). Such an absorption enhancement is much higher than those reported previously for ultrathin Si solar cells based on metal nanostructures placed on the top or at the bottom [10, 11, 14, 16-20] where either LSPR modes or SPP modes are excited alternatively. Additionally, the proposed design with the ultrathin absorber layer can reduce the usage of the active materials significantly and decrease the production costs of solar cells. Moreover, the metallic stripes on the top surface can also act as surface electrodes, eliminating the involvement of common surface electrodes (e.g., ITO). Finally, the fabrication of such metal-semiconductor-metal nanograting structures is technically feasible by using some advance nanofabrication methods $[21,22]$.

\section{Structure Design and Simulation Method}

The proposed structure with the defined structural parameters is illustrated in Figures 1(a) and 1(b), which consists from the top surface to the bottom of a periodic array of $\mathrm{Ag}$ strips, a dielectric spacer layer of $\mathrm{SiO}_{2}$, an absorber layer of Si, and an Ag nanograting substrate. The grooves of nanogating are filled with $\mathrm{SiO}_{2}$. The dielectric spacer layer can avoid the strong damping of LSPR in Ag stripes, which is responsible for enhanced light absorption inside absorber layer. Since a large spacer is very detrimental for high near-fields enhancement inside the active layer, a suitable thickness of $5 \mathrm{~nm}$ was selected here. In all our calculations, the thickness of Ag strips and the depth of nanograting are set to $t=s=40 \mathrm{~nm}$; the widths of the Ag strips and the grooves in nanograting are chosen to be $w=50 \mathrm{~nm}$ and $g=150 \mathrm{~nm}$; the thickness of Si layer and the height of Ag nanograting are fixed at $h_{1}=80 \mathrm{~nm}$ and $h_{2}=100 \mathrm{~nm}$, and the period is set to $p=300 \mathrm{~nm}$, respectively.

Numerical electromagnetic simulations were performed by the commercial finite element software of COMSOL Multiphysics 3.5. The periodic boundaries were employed to a unit cell for simulating an infinite array. Perfectly matched layers (PML) were applied in the propagation direction to eliminate the nonphysical reflections at domain boundaries. The whole structure was illuminated from the top surface at normal incidence with E-field along the metal stripes (TE wave) or H-field along the metal stripes (TM wave). The absorption inside the absorber layer for an incidence monochrome plane wave with certain wavelength was calculated by using

$$
\text { Absorption }=\oint_{s} \vec{S}(\vec{r}, \lambda) \cdot d \vec{a},
$$

where $\vec{S}(\vec{r}, \lambda)$ is the Poynting vector and $s$ is the boundary of the analyzed active layer [11].

The absorption enhancement function $(\Pi(\lambda))$ is defined as the ratio of the absorption inside the absorber layer combined with metal nanostructures to that without the metal nanostructures. The absorption efficiency for the thin film solar cell was obtained by calculating the ratio of light absorption power inside the Si layer to the incident light power. In our simulations, the absorber material is crystalline silicon and the dispersive optical properties (refractive index, absorption) of silver and crystalline silicon materials are from the experimentally obtained data $[23,24]$. The $\mathrm{SiO}_{2}$ permittivity is chosen to be 3.24 and the external dielectric environment is considered to be air. In addition, to match the solar spectrum to Si absorption, a wavelength range from $400 \mathrm{~nm}$ to $1000 \mathrm{~nm}$ was considered.

\section{Results and Discussions}

3.1. Excitations of Multiple Absorption Enhancement Bands. Figures 2(a) and 2(b) show the absorption efficiency spectra for the thin film solar cells with and without metal nanostructures, respectively. For the case of the bared $\mathrm{Si}$ film (Figure 2(a)), two absorption peaks are exhibited in the considered spectrum range. The enhanced magnetic fields inside the Si layer associated with the two absorption resonances reveal them originating from the Fabry-Periot (FP) cavity resonance [25], as the insets shown in Figure 2(a). 


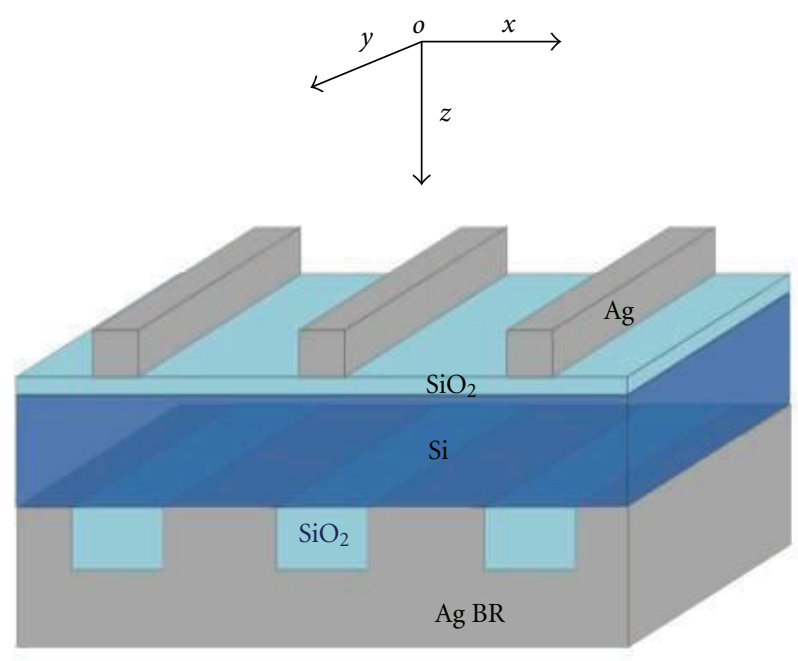

(a)

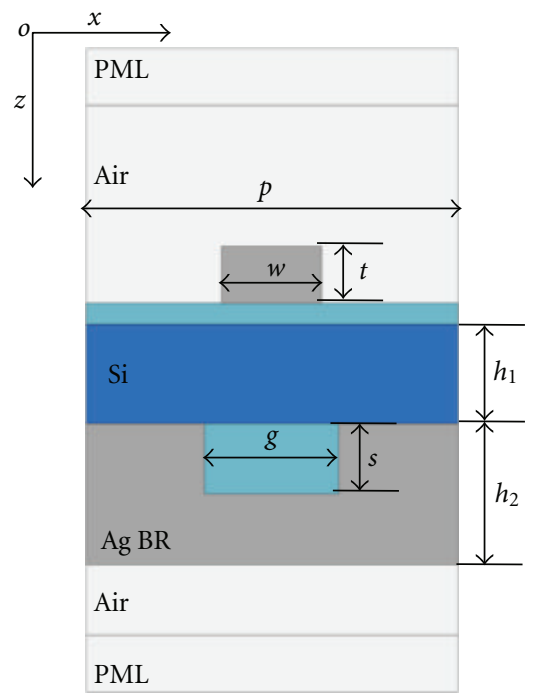

(b)

FIGURE 1: (a) Schematic diagram of the proposed thin film Si solar cell structure. (b) Side view of the unit cell, where the structural parameters are defined.

The FP resonance occurs generally when both the layer thickness and the incidence wavelengths satisfy the resonance condition, and thereby are sensitively dependent on the thickness of the Si layer. As the layer thickness increases, the FP cavity resonances shift to red and its higher-order modes will appear at the short wavelengths. When combining the Si film with the metal nanostructures, multiple absorption peaks appear in the absorption spectrum (Figure 2(b)). For comparison, the absorption enhancement spectrum $(\Pi(\lambda))$ is also calculated as shown in the insets of Figure 2(b). It can be seen clearly that the introduction of metal structures leads to multiple strong enhanced absorption bands. Furthermore, it is also indicated that generating the large absorption enhancements at the longer wavelengths where the Si film is weakly absorbing is relatively easy compared to the short wavelengths where the Si film exhibits strong absorption.

3.2. Absorption Enhancement Mechanisms. To explore the mechanisms of absorption enhancement and improve the light absorption capability of the proposed structure for solar cell applications, the absorption enhancement map $(\Pi(\lambda))$ versus the period of unit cells and the normalized magnetic field distributions $\left(\left|H_{y} / H_{0}\right|\right)$ across the Si layer at the wavelengths of the absorption enhancement peaks are investigated, as shown in Figures 3 and 4, respectively. Inside those maps of Figure 3, each point represents the fullfield simulation result with the corresponding wavelengths and periods. The field distributions $\left(\left|H_{y} / H_{0}\right|\right)$ in Figure 4 correspond to illuminations with TM polarized plane wave (H-field along the metal strips and E-field in this plane normal to the metal stripes). Four possible enhancement mechanisms, including the slab waveguide modes, the high near field enhancement associated with LSPR modes of metal strips, and the SPP modes at the metal/silicon interface as well as the coupling effects between LSPR and SPP modes will be discussed in detail in the following.

Figure 3(a) shows that the proposed structure exhibits multiple strong absorption enhancement bands at different values of periods. For the structure with a period of $320 \mathrm{~nm}$ (as the vertical white dashed line shown in Figure 3(a)), three obvious absorption enhancement peaks are observed, which are marked as "a," "b," and "c" in the map and the corresponding field distributions $\left(\left|H_{y} / H_{0}\right|\right)$ are plotted in Figures 4(a), 4(b), and 4(c), respectively. In Figure 3(a), the wavelength of the absorption peak "a" is always located around $550 \mathrm{~nm}$ when the period changes (the horizontal red dashed line). Moreover, the corresponding magnetic field distributions in Figure 4(a) exhibit a modal profile of the slab waveguide modes of finite thickness planar dielectric films, which have been given in various situations including with and without the metal back contacts $[12,26]$. The wavelength of the slab waveguide mode depends on the absorber thickness. The induced electromagnetic fields for the slab waveguide mode are mostly confined inside the absorber layer and decay sharply into the surrounding air or metal slab, contributing to the absorption enhancement.

In Figure 3(a), the absorption enhancement peak " $b$ " is always located around $700 \mathrm{~nm}$ (the black dashed line) while the absorption enhancement peak " $c$ " depends quite sensitively on the array periods (the green dashed line of " 2 "), suggesting different physical origins of the two absorption enhancement bands. Since the peak " $b$ " does not shift with changing the periods and the corresponding field distributions in Figure 4(b) carry a clear feature of localized surface plasmon mode with the enhanced magnetic fields still confined in the Si layer but mostly surrounding the metal strips, it can be attributed to LSPR mode of the metal strips. The resonant wavelength of the LSPR mode depends 


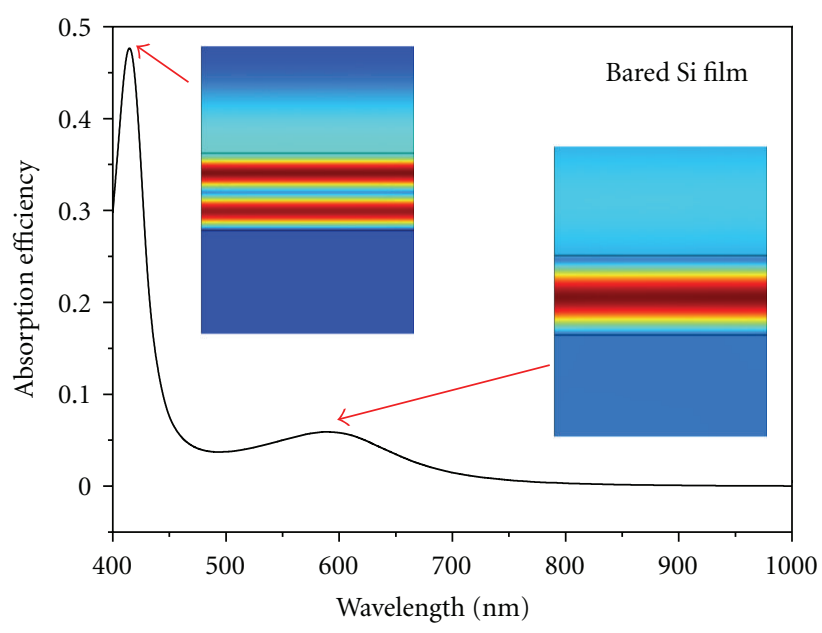

(a)

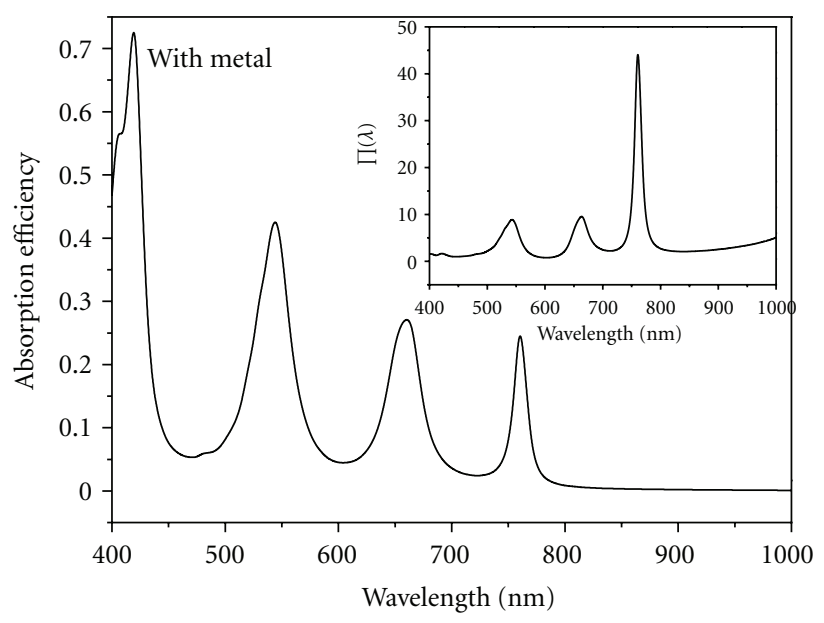

(b)

Figure 2: (a) Absorption spectrum of the bared Si film. The insets show the normalized stimulated magnetic field distributions $\left(\left|H_{y} / H_{0}\right|\right)$ at wavelengths of $420 \mathrm{~nm}$ and $600 \mathrm{~nm}$, respectively. (b) Absorption spectrum for the proposed solar cell structure under TM polarized plane wave incidences. The absorption enhancement spectrum $(\Pi(\lambda))$ is shown in the insets.

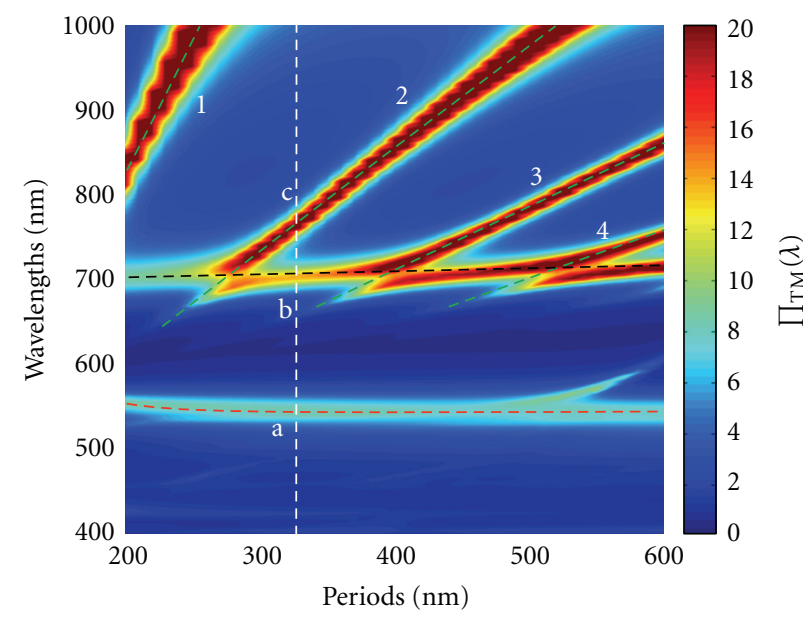

(a)

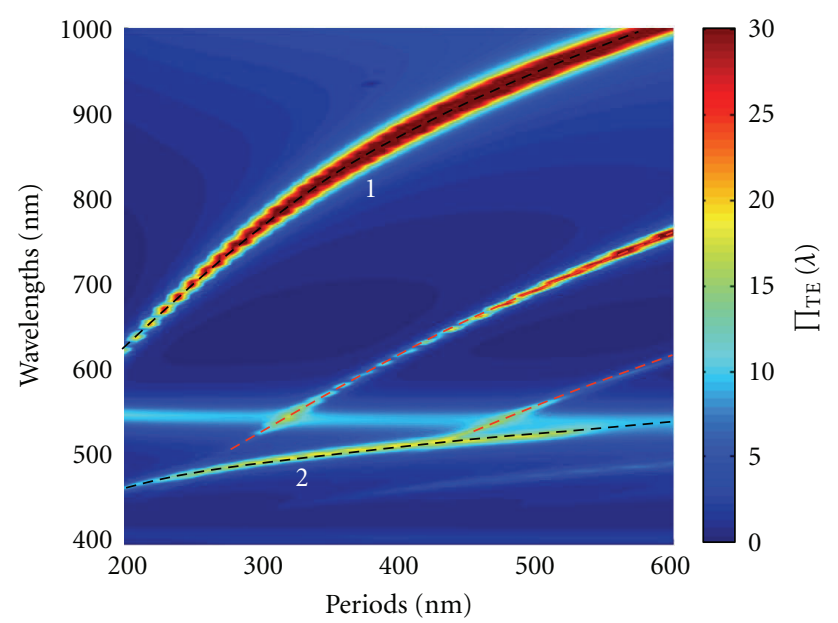

(b)

FIGURE 3: Map of the absorption enhancement $(\Pi(\lambda))$ spectra for the proposed structure with $80 \mathrm{~nm}$ thick Si layer versus the periods of unit cell under (a) TM and (b) TE polarized plane wave incidence, respectively.

sensitively on the structural parameters of metal strips. The width $w$ and thickness $t$ of metal strips have been carefully chosen through optimization because small nanoparticles scatter very weakly, whereas large nanoparticles behave like a mirror to reflect a large fraction of incident light back into free space and are not beneficial for light absorption inside the absorber layer. The peak " $\mathrm{c}$ " shifts to red with increasing the periods and the changing trend (the green dashed line of "2") presents a dispersive behavior of a SPP mode propagating at the metal/semiconductor interface according to the grating coupling theory $[27,28]$. Moreover, the corresponding field distribution in Figure 4(c) exhibits the characteristic of surface wave with the maximum field intensity near the metal/silicon interface but decay quickly apart from the interface. Thus, the peak "c" can be attributed to SPP mode excited by the bottom grating. Since the attenuation rate of the field intensity in the metal is much larger than that in the silicon layer due to higher loss of metal materials, the view of coupled light energies mostly confined into the semiconductor layer give a suggestion of absorption enhancement mechanism.

Figure 3(a) shows that as the period is increased from the initial value of $200 \mathrm{~nm}$, the wavelength of the SPP mode redshifts to approach that of the LSPR mode and a behavior of anticrossing is observed around $p=280 \mathrm{~nm}$, suggesting a strong interaction of the localized and propagating surface plasmons $[29,30]$. The absorption enhancement bands of LSPR and SPP modes become stronger and broader in 


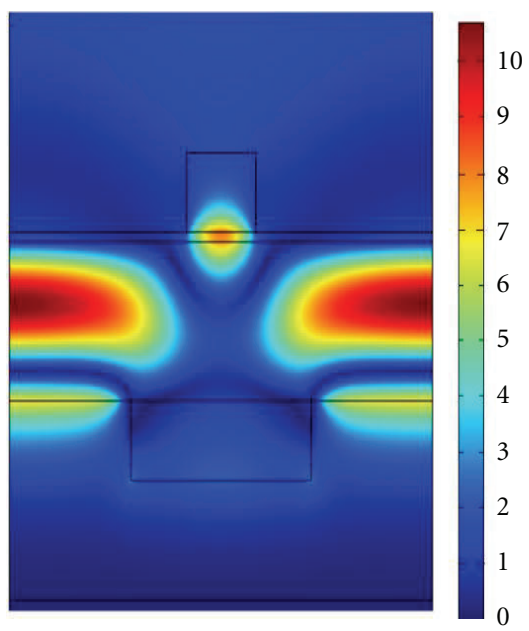

(a) $\lambda=550 \mathrm{~nm}$

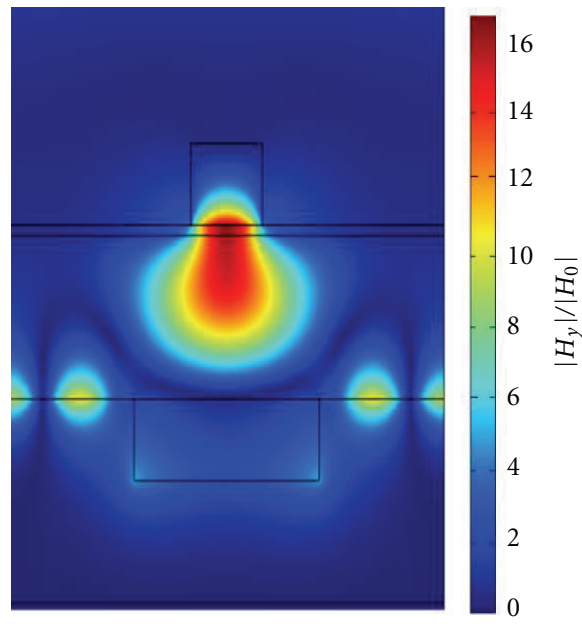

(b) $\lambda=700 \mathrm{~nm}$

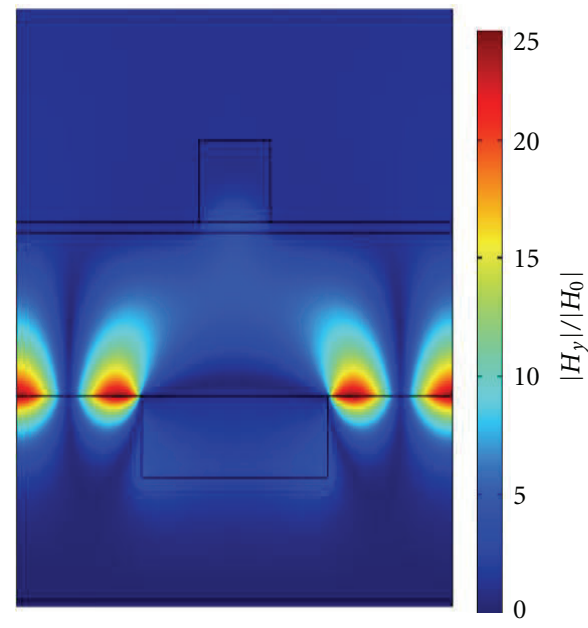

(c) $\lambda=760 \mathrm{~nm}$

FIGURE 4: The normalized magnetic field distribution $\left(\left|H_{y} / H_{0}\right|\right)$ in the cross-section for TM polarized plane wave incidence at the wavelengths of (a) $545 \mathrm{~nm}$, (b) $670 \mathrm{~nm}$, and (c) $760 \mathrm{~nm}$, corresponding to the three absorption enhancement peaks "a," "b," and "c" displayed in Figure 2(b), respectively.

the regions of coupling and light absorption is increased further by coupling effects. When the period continues to increase, multiple absorption enhancement bands appear and the anticrossing behavior is observed when the wavelengths of these absorption peaks overlap with that of LSPR mode. The changing trend of other three enhanced absorption bands with the periods are highlighted by the green dashed lines of " 1, ," " 3 ," and " 4 ," respectively, which present a similar dispersive behavior as that of the SPP mode marked by "2," implying a SPP-related absorption enhancement mechanism. Obviously, as the period increases, the SPP mode redshifts and more higher-order SPP modes appear, leading to multiple absorption enhancement bands at longer wavelengths.

Unlike TM polarizations, the SPP and LSPR modes are absent under TE plane wave incidence (E-field along the metal strips and $\mathrm{H}$-field in this plane normal to the metal strips). Only the enhanced absorption related to the TE waveguide modes are observed in Figure 4(b), where the two enhanced absorption bands marked as the black dashed line of " 1 " and "2" are expected to attribute to the $\mathrm{TE}_{0}$ and $\mathrm{TE}_{1}$ waveguide modes. It can be seen that the absorption enhancement factor related to the TE waveguide modes is very high, but the width of absorption enhancement bands is very narrow. As the period become large, the absorption enhancement increases and some higher-order modes of $\mathrm{TE}_{0}$ waveguide appear (the red dashed line), contributing to the overall absorption enhancement under TE plane wave incidence.

3.3. Total Absorption Enhancement under the Solar Illuminations. To perform a full evaluation on the performance of the proposed design, the total absorption enhancement under solar illuminations must be considered. Generally, the short circuit current in a solar cell is proportional to the number of absorbed photons if assuming unity internal 

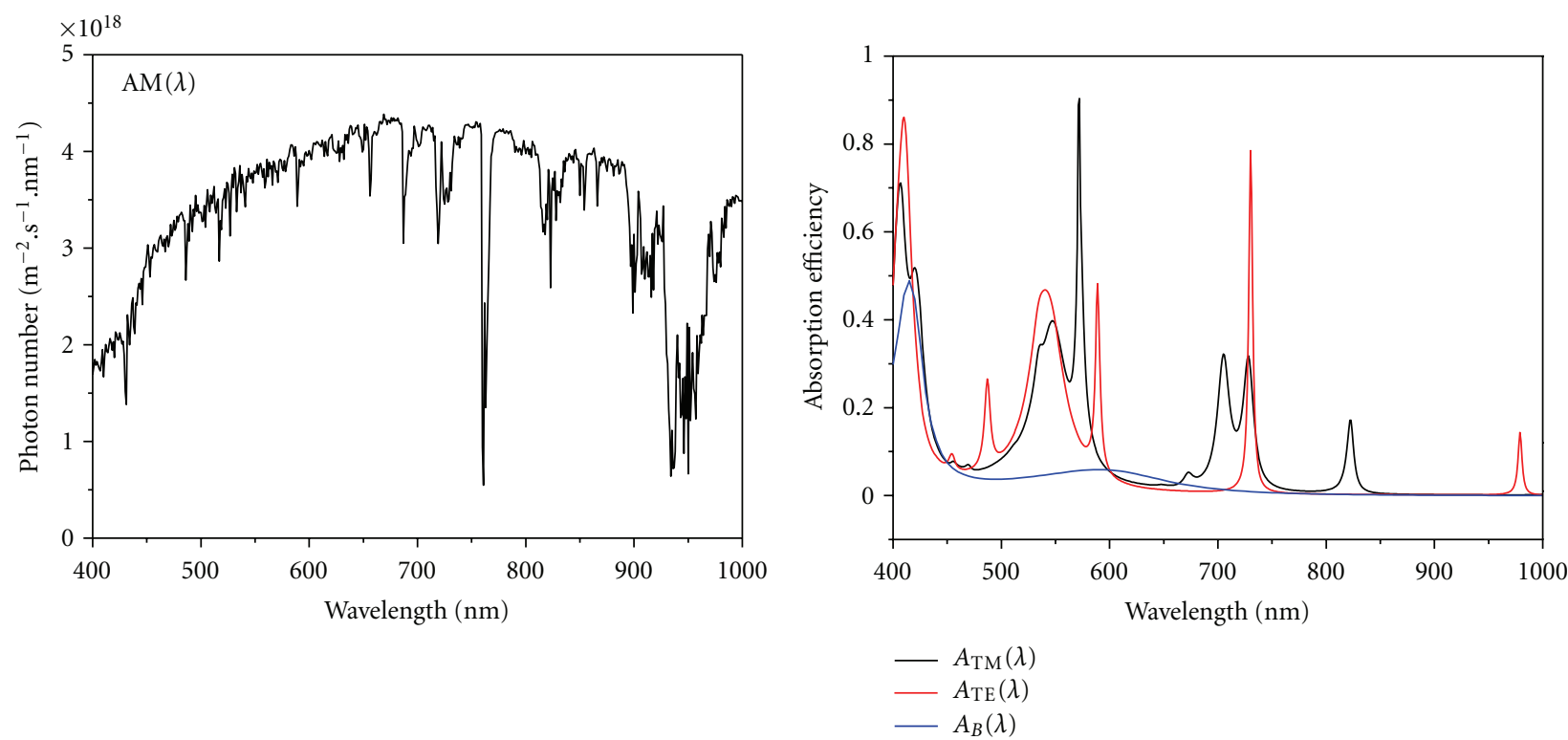

(a)

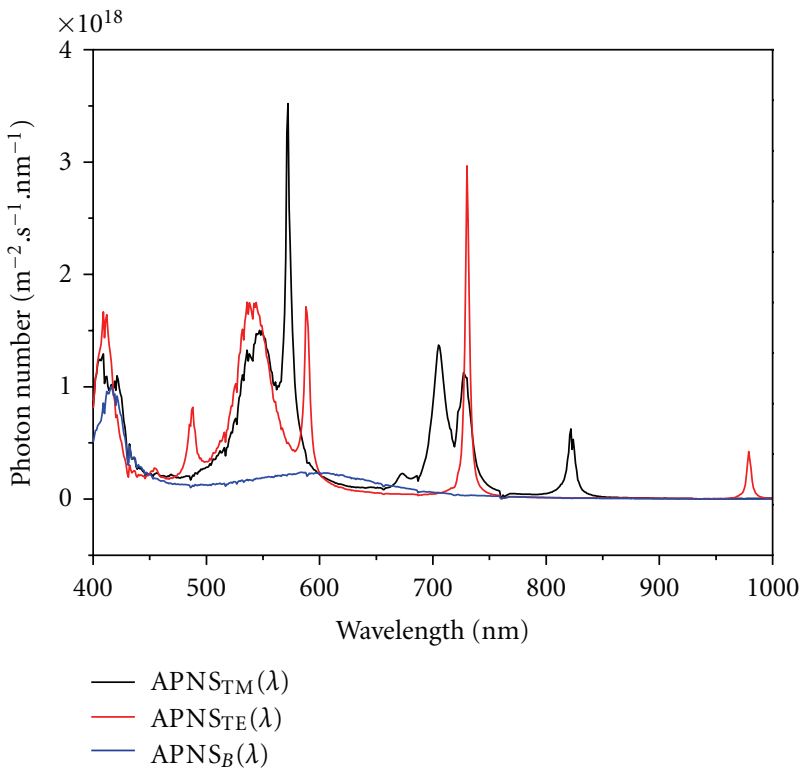

(c) (b)

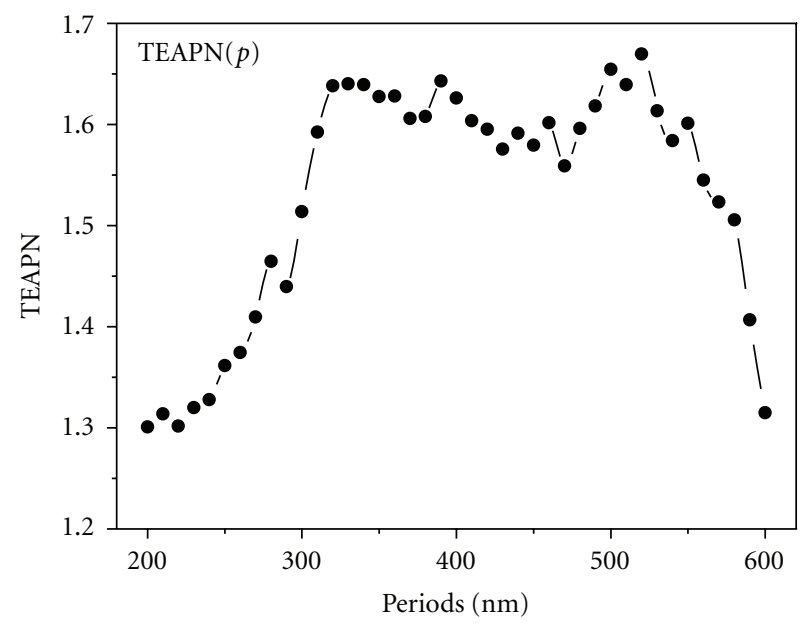

(d)

FIgURe 5: (a) Photon number spectrum of the AM1.5 solar irradiations $(\operatorname{AM}(\lambda))$. (b) Absorption efficiency spectrum $(A(\lambda))$. (c) Absorbed photon number spectrum (APNS). (d) Total enhancement of absorbed photon number (TEAPN) inside the Si layer over the entire solar spectrum versus the different array periods. The subscripts "TE" and "TM" in (a) and (b) represent different polarizations for the proposed solar cells with a period of $550 \mathrm{~nm}$. The subscript " $B$ " corresponds to the bared Si film with a thickness of $80 \mathrm{~nm}$.

quantum efficiency (every photoexcited electron-hole pair is collected). Therefore, the total absorption enhancement must be evaluated based on the number of photons being absorbed. Figure 5(a) presents the photon number spectrum of the standard AM1.5 solar irradiation $(\operatorname{AM}(\lambda))$. The absorbed photon number spectrum (APNS) inside the active layer under solar illuminations can be obtained by using the following equation [31]:

$$
\operatorname{APNS}(\lambda)=A(\lambda) \times \operatorname{AM}(\lambda)
$$

where the absorption efficiency spectrum $A(\lambda)$ represents the absorbed power inside the $\mathrm{Si}$ layer with unity incident light power, as shown in Figure 5(b) for TM or TE polarized light incidence. Figure 5(c) displays the APNS for the proposed solar cells at two polarizations and that for an $80 \mathrm{~nm}$ thick bared Si film, respectively. Compared to the bared Si film, the introduction of metal structures in thin film solar cells leads to an obvious increase of the absorbed photon numbers inside the Si layer. 
Considering the equal contributions from TE and TM polarized light, the APNS of the proposed solar cells under randomly polarized sunlight can be described as

$$
\operatorname{APNS}(\lambda)=\frac{1}{2}\left(\operatorname{APNS}_{\mathrm{TE}}(\lambda)+\operatorname{APNS}_{\mathrm{TM}}(\lambda)\right)
$$

where the subscript represent the case of TE or TM polarizations. The total enhancement of absorbed photon number (TEAPN) inside the Si layer is defined as

$$
\operatorname{TEAPN}=\frac{\int \operatorname{APNS}(\lambda)_{\text {with_metal-structures }} d \lambda-\int \operatorname{APNS}(\lambda)_{\text {without_metal-structures }} d \lambda}{\int \operatorname{APNS}(\lambda)_{\text {without_metal-structures }} d \lambda} .
$$

The wavelength range for integrating is selected from $400 \mathrm{~nm}$ to $1000 \mathrm{~nm}$. Figure $5(\mathrm{~d})$ shows the simulation results of the total enhancement (TEAPN) versus different array periods. Obviously, a very large enhancement up to $170 \%$ can be achieved in our designed structures when compared with the case of a bared Si layer. Furthermore, the high enhancement of about $170 \%$ is broadband for the array period with a large range from about $300 \mathrm{~nm}$ to $550 \mathrm{~nm}$, thus providing a very good fabrication tolerance for practical devices. Such results are impressive and superior to those of some previous designs of plasmonic thin film solar cells. In addition, it should be pointed that although the thickness of $80 \mathrm{~nm}$ employed in our simulations was used in certain devices, the active layer thickness for the ultrathin Si solar cells is typically $200-600 \mathrm{~nm}$ in the practical device applications. In this case, more optical waveguide modes would be excited to play important roles in absorption enhancement $[11,12]$. Therefore, for the proposed structure with thicker active layer, multiple plasmon resonance modes associated with the introduction of metal structures together with these optical modes are predicated to contribute to the overall absorption enhancement. Since the configuration of metallic materials rather than the discussed crystalline silicon materials is responsible for the absorption enhancement in our proposed design, similar performances are also expected by extending the similar design into other active solar materials such as CdTe and organics. Therefore, our proposed design has the potential to become a solar cell platform for various thin film solar cell systems.

\section{Conclusion}

In conclusion, the light absorption properties of a novel ultrathin film Si solar cell structure were discussed in detail by using the finite element method. As compared to a bared $\mathrm{Si}$ thin film, the total absorption enhancement of absorbed photon number can reach to $170 \%$ within a broad period range by introducing $\mathrm{Ag}$ strips on top and $\mathrm{Ag}$ nanograting as the back contacts in the solar cell structure. The absorption enhancement mechanisms are revealed by analyzing the enhanced field distributions and investigating the absorption enhancement spectra as a function of periods. These results pave a promising way for the realization of high efficiency thin-film solar cells.

\section{Acknowledgments}

This work is supported by the National Science Foundation of China (no. 10974183 and 11104252), the Ministry of Education of China (no. 20114101110003), the Aeronautical Science Foundation of China (no. 2011ZF55015), and the Basic and Frontier Technology Research Program of Henan Province (no. 122300410162 and 112300410264).

\section{References}

[1] M. A. Green, "Lambertian light trapping in textured solar cells and light-emitting diodes: analytical solutions," Progress in Photovoltaics, vol. 10, no. 4, pp. 235-241, 2002.

[2] H. A. Atwater and A. Polman, "Plasmonics for improved photovoltaic devices," Nature Materials, vol. 9, no. 3, pp. 205213, 2010.

[3] K. R. Catchpole and A. Polman, "Plasmonic solar cells," Optics Express, vol. 16, no. 26, pp. 21793-21800, 2008.

[4] P. Spinelli, V. Ferry, J. van de Groep et al., "Plasmonic light trapping in thin-film Si solar cells," Journal of Optics, vol. 14, no. 2, Article ID 024002, 11 pages, 2012.

[5] D. M. Schaadt, B. Feng, and E. T. Yu, "Enhanced semiconductor optical absorption via surface plasmon excitation in metal nanoparticles," Applied Physics Letters, vol. 86, no. 6, Article ID 063106, 3 pages, 2005.

[6] D. Qu, F. Liu, Y. Huang, W. Xie, and Q. Xu, "Mechanism of optical absorption enhancement in thin film organic solar cells with plasmonic metal nanoparticles," Optics Express, vol. 19, no. 24, pp. 24795-24803, 2011.

[7] C. Hägglund, M. Zäch, G. Petersson, and B. Kasemo, "Electromagnetic coupling of light into a silicon solar cell by nanodisk plasmons," Applied Physics Letters, vol. 92, no. 5, Article ID 053110, 3 pages, 2008.

[8] S. Pillai, K. R. Catchpole, T. Trupke, and M. A. Green, "Surface plasmon enhanced silicon solar cells," Journal of Applied Physics, vol. 101, no. 9, Article ID 093105, 8 pages, 2007.

[9] K. R. Catchpole and A. Polman, "Design principles for particle plasmon enhanced solar cells," Applied Physics Letters, vol. 93, no. 19, Article ID 191113, 3 pages, 2008.

[10] R. A. Pala, J. White, E. Barnard, J. Liu, and M. L. Brongersma, "Design of plasmonic thin-film solar cells with broadband absorption enhancements," Advanced Materials, vol. 21, no. 34, pp. 3504-3509, 2009.

[11] W. Wang, S. Wu, K. Reinhardt, Y. Lu, and S. Chen, "Broadband light absorption enhancement in thin-film silicon solar cells," Nano Letters, vol. 10, no. 6, pp. 2012-2018, 2010.

[12] V. E. Ferry, L. A. Sweatlock, D. Pacifici, and H. A. Atwater, "Plasmonic nanostructure design for efficient light coupling 
into solar cells," Nano Letters, vol. 8, no. 12, pp. 4391-4397, 2008.

[13] R. Biswas and D. Zhou, "Simulation and modelling of photonic and plasmonic crystal back reflectors for efficient light trapping," Physica Status Solidi A, vol. 207, no. 3, pp. 667$670,2010$.

[14] R. B. Dunbar, T. Pfadler, and L. Schmidt-Mende, "Highly absorbing solar cells - a survey of plasmonic nanostructures," Optics Express, vol. 20, no. 2, pp. A177-A189, 2012.

[15] W. Wang, S. Wu, R. J. Knize, K. Reinhardt, Y. Lu, and S. Chen, "Enhanced photon absorption and carrier generation in nanowire solar cells," Optics Express, vol. 20, no. 4, pp. 3733 3743, 2012.

[16] C. Rockstuhl, S. Fahr, and F. Lederer, "Absorption enhancement in solar cells by localized plasmon polaritons," Journal of Applied Physics, vol. 104, no. 12, Article ID 123102, 7 pages, 2008.

[17] Y. A. Akimov, W. S. Koh, and K. Ostrikov, "Enhancement of optical absorption in thin-film solar cells through the excitation of higher-order nanoparticle plasmon modes," Optics Express, vol. 17, no. 12, pp. 10195-10205, 2009.

[18] H. Shen, P. Bienstman, and B. Maes, "Plasmonic absorption enhancement in organic solar cells with thin active layers," Journal of Applied Physics, vol. 106, no. 7, Article ID 073109, 5 pages, 2009.

[19] W. Bai, Q. Gan, G. Song, L. Chen, Z. Kafafi, and F. Bartoli, "Broadband short-range surface plasmon structures for absorption enhancement in organic photovoltaics," Optics Express, vol. 18, no. 4, pp. A620-A630, 2010.

[20] E. Battal, T. A. Yogurt, L. E. Aygun, and A. K. Okyay, “Triangular metallic gratings for large absorption enhancement in thin film Si solar cells," Optics Express, vol. 20, no. 9, pp. 9548-9464, 2009.

[21] L. J. Guo, "Nanoimprint lithography: methods and material requirements," Advanced Materials, vol. 19, no. 4, pp. 495-513, 2007.

[22] J. Y. Chen and K. W. Sun, "Enhancement of the light conversion efficiency of silicon solar cells by using nanoimprint antireflection layer," Solar Energy Materials and Solar Cells, vol. 94, no. 3, pp. 629-633, 2010.

[23] P. B. Johnson and R. W. Christy, "Optical constants of the noble metals," Physical Review B, vol. 6, no. 12, pp. 4370-4379, 1972.

[24] E. D. Palik, Handbook of Optical Constants of Solids, Academic Press, 1985.

[25] A. W. Snyder and J. D. Love, Optical Waveguide Theory, Springer, 1983.

[26] V. E. Ferry, J. N. Munday, and H. A. Atwater, "Design considerations for plasmonic photovoltaics," Advanced Materials, vol. 22, no. 43, pp. 4794-4808, 2010.

[27] H. Raether, Surface Plasmons on Smooth and Rough Surfaces and on Gratings, Springer, Berlin, Germany, 1988.

[28] N. Papanikolaou, "Optical properties of metallic nanoparticle arrays on a thin metallic film," Physical Review B, vol. 75, no. 23, Article ID 235426, 7 pages, 2007.

[29] J. N. He, G. W. Cai, P. Ding, C. Z. Fan, and E. J. Liang, "Surface plasmons coupling for local field enhancement in nanoring arrays on a metallic film," Physics Express, vol. 2, no. 10, 2012.

[30] P. Ding, E. J. Liang, G. W. Cai, W. Q. Hu, C. Z. Fan, and Q. Z. Xue, "Dual-band perfect absorption and field enhancement by interaction between localized and propagating surface plasmons in optical metamaterials," Journal of Optics, vol. 13, no. 7, Article ID 075005, 7 pages, 2011.
[31] Y. A. Akimov and W. S. Koh, "Design of plasmonic nanoparticles for efficient subwavelength light trapping in thin-film solar cells," Plasmonics, vol. 6, no. 1, pp. 155-161, 2011. 

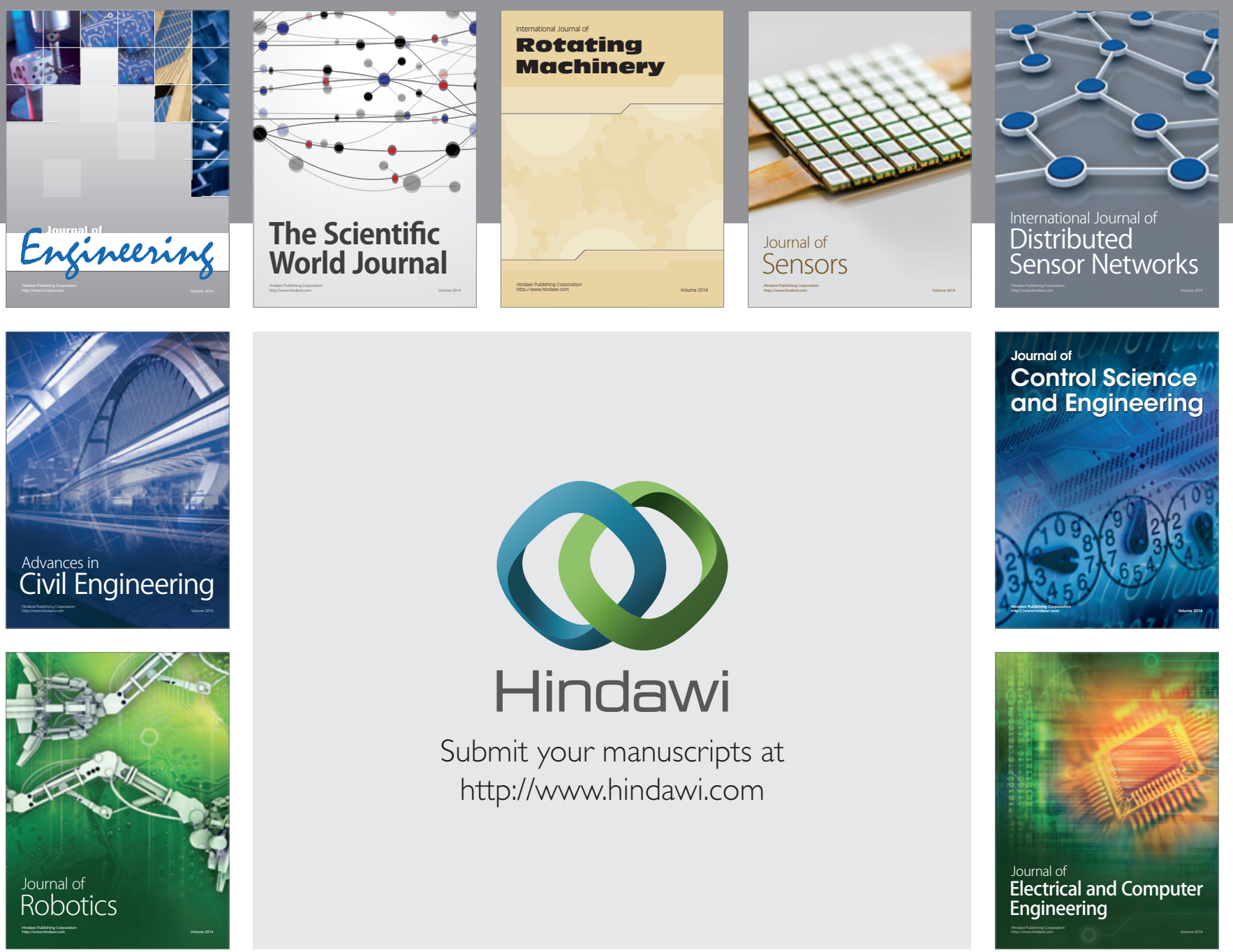

Submit your manuscripts at

http://www.hindawi.com
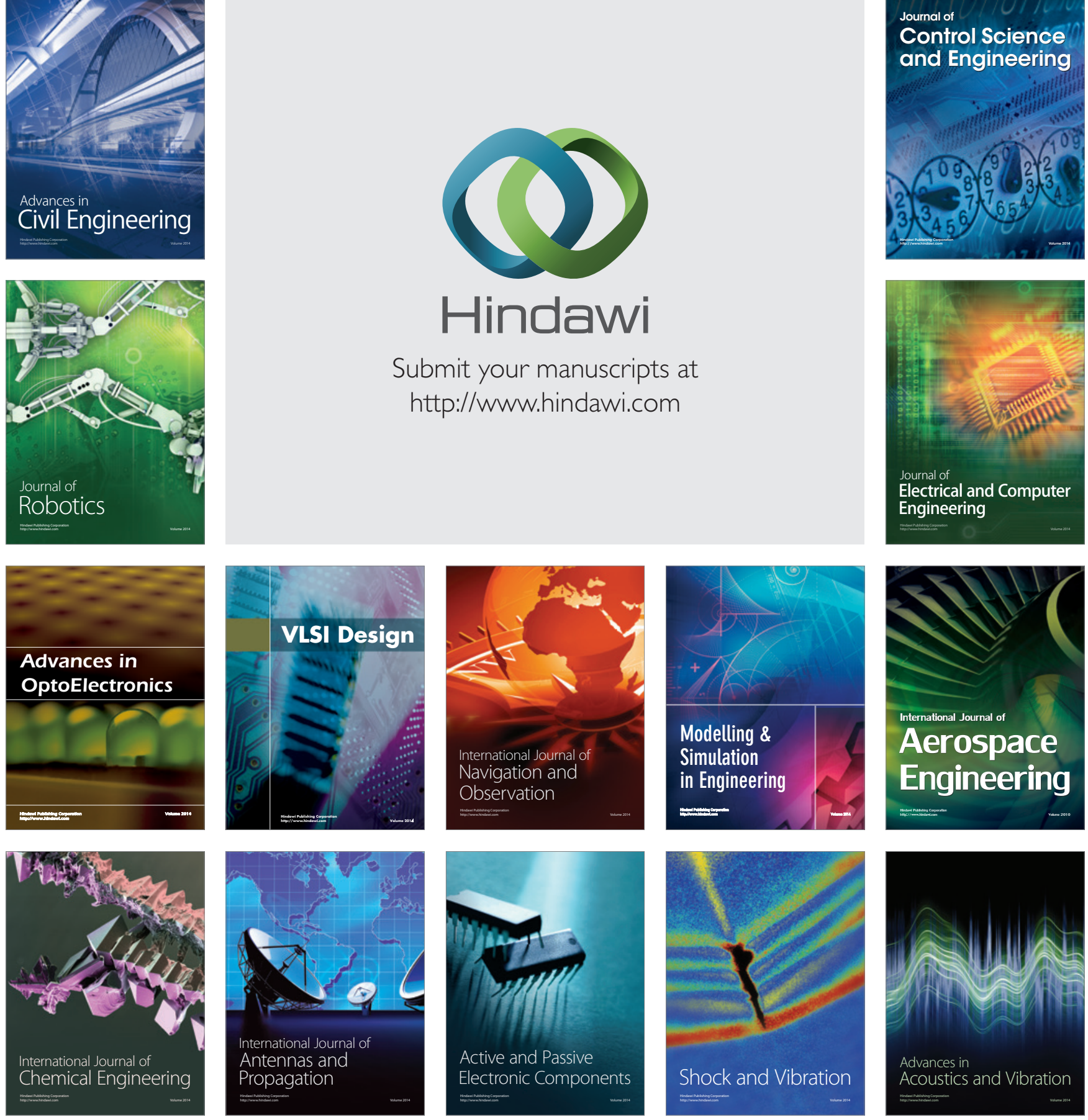